\title{
Relationships between users, resources and services in learning object repositories
}

\author{
Jordi Conesa, Julià Minguillón, and M. Elena Rodríguez \\ Universitat Oberta de Catalunya \\ Rambla Poblenou 156, 08018 Barcelona, Spain
}

\begin{abstract}
In this paper we describe a proposal for defining the relationships between resources, users and services in a digital repository. Nowadays, virtual learning environments are widely used but digital repositories are not fully integrated yet into the learning process. Our final goal is to provide final users with recommendation systems and reputation schemes that help them to build a true learning community around the institutional repository, taking into account their educational context (i.e. the courses they are enrolled into) and their activity (i.e. system usage by their classmates and teachers). In order to do so, we extend the basic resource concept in a traditional digital repository by adding all the educational context and other elements from end-users' profiles, thus bridging users, resources and services, and shifting from a library-centered paradigm to a learning-centered one.
\end{abstract}

Keywords: Digital repositories, Learning objects, Resources, Modeling, Recommendation systems, Reputation schemes

\section{Introduction}

Learning object repositories are becoming more and more popular as more and more educational institutions are widening the scope of their institutional repositories, including not only research outcomes but also resources created during the teaching and learning processes [6]. Nevertheless, it is well known that final users (mostly teachers and learners) do not use repositories $[1,6]$, mainly because these systems have been designed and implemented without taking into account end-users [12]. In order to become true learning spaces, learning object repositories need to attract users by creating true learning communities $[9,2]$. In [7] the authors describe a possible set of additional services that could be built on top of institutional repositories in order to increase their usage, pursuing also the creation of a community of learning.

A preliminary study on the UOC institutional repository (namely $\mathrm{O} 2^{1}$ ), based on interviews with end-users (teachers and learners), showed that rating, tagging and finding related resources were the most valued services. Therefore, as part of the MAVSEL Project ${ }^{2}$, we intend to extend the default set of services

\footnotetext{
${ }^{1}$ O2: http://openaccess.uoc.edu/webapps/o2/?locale=en

2 Project MAVSEL: http://www.ieru.org/projects/mavsel/index.html
} 
available in the UOC institutional repository. In order to do so, we propose to establish a conceptual model involving users, resources, additional services and their educational context. Once this initial model has been defined, a reputation scheme for both users and resources is outlined, in order to provide the basis for building a recommendation system and a service-oriented repository, taking into account user's needs and educational context.

This paper is structured as follows: Section 2 describes the main drawbacks of using digital repositories as the center of a community of learning due to their intrinsic nature. In Section 3 we propose the information architecture model involving users, resources and services. The basics of the reputation schemes and the recommendation system based on such proposal are presented in Section 4. Finally, the main results of this work are summarized in Section 5.

\section{Learning object repositories}

Digital repositories have been traditionally designed and implemented by librarians with the help of IT staff. Out-of-the-box solutions such as DSpace ${ }^{3}$ have allowed educational institutions to build institutional repositories but from a top-down approach, without taking into account end-users' needs. Furthermore, using institutional repositories to store and share learning objects has some additional drawbacks. For instance, not all learning objects can be fully described using only author, title, publication date and some keywords. From a teacher's perspective, it is also important to contextualize every didactic resource according to the specific particularities of the teaching process. This suggests that learning objects need to be properly modeled from several related perspectives. In [8] different context categories for learning objects are identified, namely thematic context (which describes the learning objects content, i.e. domain information), pedagogical context (which deals with the knowledge and information about the teaching-learning processes where the learning objects are used), the learner context (which describes the characteristics of the students who are expected to use the learning objects), organizational context (which covers the structural composition and sequencing of learning objects) and the historical/statistical context (which captures information regarding the social patterns that are derived from the learning objects usage). The required metadata to capture all previous information mainly has, according to [10], an extrinsic nature, i.e. the values of the metadata characterizing context categories (except for the thematic context) change depending on the educational context where the learning objects are used. The problem is that available solutions to build institutional repositories mainly deal with intrinsic metadata, i.e. metadata whose values remain immutable (author and title are examples of intrinsic metadata) in all possible educational context of the learning objects. Specifically, this is the case of DSpace which uses Dublin Core as metadata schema for describing learning objects. One of the decisions made during the conceptualization of Dublin Core was precisely to avoid the definition of extrinsic metadata ([10]).

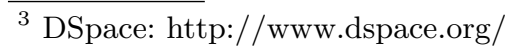




\section{A Model to Specify the Context of Learning Resources}

As aforesaid, one of the main problems of learning object repositories is its lack of integration within the learning environment where they are used. In the context of learning, learning resources are used by human agents within a context (a subject, for instance) and for a given purpose (for giving support to an exercise, as a lecture, etc.). Relating learning resources to their learning context will facilitate the creation of services that use such contextual information to behave more intelligently. Some examples of such services may be recommendation systems or automatic evaluators. With this objective in mind, the conceptual schema in Figure 1 shows a possible contextualization of the learning domain where the learning resources are used. As can be seen in Figure 1, learning resources should be related to some concepts of the learning management system (LMS), which come from a package called LMS. Our proposal takes into account the offered curricula, the subjects each curriculum contains, the knowledge areas related to each subject, the human agents of the LMS and, in the case of learners, their enrolled and passed subjects.

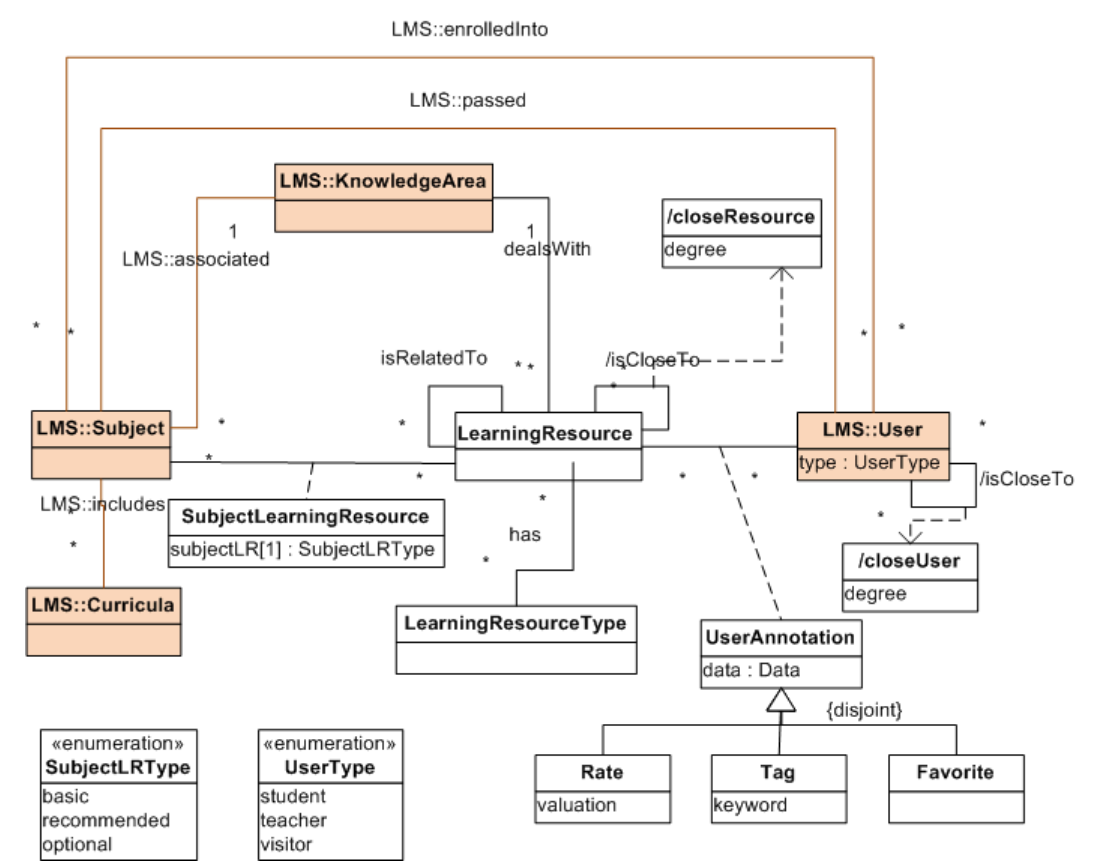

Fig. 1. Conceptualization of the learning resource context.

The main element of the proposed conceptual schema is Learning Resource. This concept represents the learning resources contained in the repository. Each learning resource is related with the knowledge area it deals with, representing 
the thematic context of learning resources. Learning resources may also have a type, which can define their kind from a pedagogical point of view (exercise, lecture...) or their format (in this case, learning resources are described according to how they are perceived by users). In addition, learning objects can be related with other learning objects. Learning object relations are denoted in Figure 1 by means of the generic relationship isRelatedTo. Examples of learning object relations could be those proposed by Dublin Core and available in DSpace (e.g. references, requires, or is version of) as well as other relations, relevant from a pedagogical point of view (for example exemplifies, deepens or summarizes) that have been proposed by several authors $([3-5,11])$. Both learning resource type characterization and relations between learning objects partially describe the pedagogical context of the learning resources. The pedagogical context of learning objects is complemented by adding the subjects where each learning object is used and through the inclusion of information that records whether the resource is mandatory, recommended or optional. This information will help to estimate the relevance of resources for each user in a given subject. On the other hand, the student context is described (at a minimum detail level) by means of the subjects that he or she has enrolled and passed. Finally, users can perform different kinds of annotations on the learning resources. In the proposed schema, the allowed user annotations include rate a resource, tag a resource or make it favorite. Annotations on learning objects represent historical/statistical context and can be considered a first step towards creating a community around learning resources and subjects.

As aforementioned, the metadata schema proposed by DSpace uses Dublin Core. Only a few relationships of those proposed in Figure 1 can be represented by extending the Dublin Core metadata elements supported by DSpace. In addition, it is important to note that Dublin Core relationships are not able to fully describe all the semantic richness of relevant relationships [11]. The nonqualified metadata elements (or their refinements in some cases) that can be used are DC.Subject, DC.Relation and DC.Type: DC Subject allows to model the knowledge area the learning resources deal with; DC.Relation relates relationships between different learning resources; and DC.Type defines the type of learning resource (only representational aspects). The remaining concepts and relationships presented in Figure 1 can not be captured given the intrinsic nature of Dublin Core. Another relevant information is to determine how close two resources and/or users are (i.e. relationship isCloseTo as Figure 1 shows). Closeness has been defined as derived association classes. Instances of such classes should be calculated differently in each environment and depending on what services are implemented in each case.

\section{Recommendation system and reputation schemes}

Currently now, digital repositories based on DSpace show the last five items published in the repository, which are supposed to be interesting for most users. Using the relationships aforementioned, we want to change such criterion (i.e. 
publication date) to other more appropriate in the context of a virtual learning environment, taking into account the activity of the community of learning. Once the proposed layer of additional services [7] will be running on top of the institutional repository, we will be able to gather the following data:

- With respect to resources: the number of times a resource has been visited, downloaded, rated (and the individual ratings given by registered users), favorited and tagged (and the individual tags).

- With respect to users: the number of resources she has downloaded, rated (and her ratings), favorited, tagged (and her tags).

With all this data we will be able to compute the following distance measures for both resources and users:

- For each resource $R_{i}$ : a reputation scheme $F_{R}\left(R_{i}\right)$ used to rank resources according to their "popularity".

- For each pair of resources $R_{i}, R_{j}$ : a distance function $d_{R}\left(R_{i}, R_{j}\right)$ used to find the "closest" resources.

- For each user $U_{i}$ : a reputation scheme $F_{U}\left(U_{i}\right)$ used to rank users according to their "activity".

- For each pair of users $U_{i}, U_{j}$ : a distance function $D_{U}\left(U_{i}, U_{j}\right)$ used to find the most "similar" users, i.e. those with the same interests.

- For each pair user/resource $U_{i}, R_{j}$ : a distance function $D_{U, R}\left(U_{i}, R_{j}\right)$ used to find the most "relevant" resource for a given user. It can be also used to find the most "potential" users for a given resource.

The concepts of "popularity", "activity", etc. may then be tailored to the particular needs of the learning process, so learners are able to find and navigate through the most valuable resources taking into account all their needs as well as their context. For instance, at the beginning of the academic semester, some resources (i.e. preliminary readings) can be defined as more "popular" than others by just including such time concept in $F_{R}$.

\section{Conclusions}

In this paper we have conceptualized the relationships between users, resources and services in a digital repository, with the aim of better supporting the activities of searching and browsing learning resources, taking into account the educational context. With such proposal, the digital repository becomes more integrated into the learning process, and no longer is a mere space where thousands of resources can be found. Learners can find "close" resources according to their needs. We have also outlined the underlying reputation schemes for ranking users and resources, so the recommendation system will be able to provide users with the most tailored resources, according to their context.

Current and future work in this subject includes the development of the aforementioned services into the UOC institutional repository, gathering real 
usage data during one or more academic semesters. Data will be used to finetune both the recommendation system and the reputation schemes, the previous step before building a contextualized searching/browsing engine on top of the institutional repository giving support to the learning community.

\section{Acknowledgements}

This paper has been partially supported by Spanish Ministry of Science and Innovation funded Project MAVSEL (ref. TIN2010-21715-C02-02).

\section{References}

1. Caris, M.A.: Why don't faculty use learning object repositories? In: Cantoni, L., McLoughlin, C. (eds.) Proceedings of World Conference on Educational Multimedia, Hypermedia and Telecommunications 2004. pp. 2838-2840. AACE, Lugano, Switzerland (2004), http://www.editlib.org/p/12413

2. Churchill, D., Wong, W., Law, N., Salter, D., Tai, B.: Social BookmarkingRepository-Networking: Possibilities for Support of Teaching and Learning in Higher Education. SERIALS REVIEW 35(3), 142-148 (SEP 2009)

3. Fischer, S.: Course and exercise sequencing using metadata in adaptative hypermedia learning systems. ACM Journal of Educational Resources in Computing 1(1) (2001)

4. Hamel, C.J., Ryan-Jones, D.: Towards a comprehensive learning object metadata: Incorporation of context to stipulate meaningful learning and enhance learning object reusability. International Journal of Educational Technology 3(1) (2002)

5. Lu, E.J.L., Hsieh, C.J.: A relation metadata extension for SCORM content aggregation model. Computer Standards \& Interfaces 31, 1028-1035 (2009)

6. Margaryan, A., Littlejohn, A.: Repositories and communities at cross-purposes: issues in sharing and reuse of digital learning resources. JOURNAL OF COMPUTER ASSISTED LEARNING 24(4), 333-347 (AUG 2008)

7. Minguillón, J., Rodríguez, M.E., Conesa, J.: Extending learning objects by means of social networking. In: Luo, X., Spaniol, M., Wang, L., Li, Q., Nejdl, W., Zhang, W. (eds.) ICWL. Lecture Notes in Computer Science, vol. 6483, pp. 220-229. Springer (2010)

8. Mohammed, P., Mohan, P.: Contextualizing learning objects using ontologies. Computational Intelligence 23(3), 339-355 (2007)

9. Monge, S., Ovelar, R., Azpeitia, I.: Repository 2. 0: Social dynamics to support community building in learning object repositories. Social Dynamics 4, 191-204 (2008), http://www.ijello.org/Volume4/IJELLOv4p191-204Monge.pdf

10. Recker, M., Wiley, D.A.: A non-authoritative educational metadata ontology for filtering and recommending learning objects. Journal of interactive learning environments 9(3), 255-271 (2001)

11. Rodriguez, M.E., Conesa, J., Sicilia, M.A.: Clarifying the Semantics of Relationships between Learning Objects. In: Sartori, F and Sicilia, MA and Manouselia, K (ed.) METADATA AND SEMANTIC RESEARCH, PROCEEDINGS. Communications in Computer and Information Science, vol. 46, pp. 35-47. Dept Comp Sci, Syst \& Commun; Univ Milan-Bicocca (2009)

12. St Jean, B., Rieh, S.Y., Yakel, E., Markey, K.: Unheard Voices: Institutional Repository End-Users. COLLEGE \& RESEARCH LIBRARIES 72(1), 21-42 (JAN 2011) 